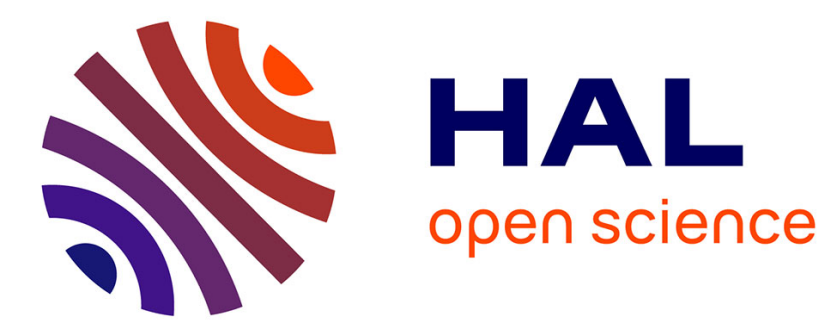

\title{
Sharing object models for multi-modality medical image simulation: a semantic approach
}

\author{
G. Forestier, A. Marion, H. Benoit-Cattin, P. Clarysse, D. Friboulet, T \\ Glatard, P. Hugonnard, C. Lartizien, H. Liebgott, J. Tabary, et al.
}

\section{- To cite this version:}

G. Forestier, A. Marion, H. Benoit-Cattin, P. Clarysse, D. Friboulet, et al.. Sharing object models for multi-modality medical image simulation: a semantic approach. International Symposium on Computer-Based Medical Systems (CBMS), 2011, Bristol, UK, United Kingdom. pp.1-6, 10.1109/CBMS.2011.5999167 . hal-01922169

\section{HAL Id: hal-01922169 \\ https://hal.science/hal-01922169}

Submitted on 15 Nov 2019

HAL is a multi-disciplinary open access archive for the deposit and dissemination of scientific research documents, whether they are published or not. The documents may come from teaching and research institutions in France or abroad, or from public or private research centers.
L'archive ouverte pluridisciplinaire HAL, est destinée au dépôt et à la diffusion de documents scientifiques de niveau recherche, publiés ou non, émanant des établissements d'enseignement et de recherche français ou étrangers, des laboratoires publics ou privés. 


\title{
Sharing object models for multi-modality medical image simulation: a semantic approach
}

\author{
Germain Forestier $^{1}$, Adrien Marion ${ }^{2}$, Hugues Benoit-Cattin ${ }^{2}$, Patrick Clarysse ${ }^{2}$ \\ Denis Friboulet $^{2}$, Tristan Glatard ${ }^{2}$, Patrick Hugonnard ${ }^{3}$, Carole Lartizien ${ }^{2}$ \\ Hervé Liebgott ${ }^{2}$, Joachim Tabary ${ }^{3}$, Bernard Gibaud ${ }^{1}$ \\ ${ }^{1}$ INSERM / INRIA / CNRS / Univ. Rennes 1, VISAGES U746, Rennes, France \\ ${ }^{2}$ Université de Lyon, CREATIS ; CNRS UMR5220 ; Inserm U1044 ; INSA-Lyon ; Université Lyon 1, France \\ ${ }^{3}$ CEA-LETI-MINATEC, Recherche technologique, 17 Rue des martyrs, 38054 Grenoble Cedex 09, France
}

\begin{abstract}
Medical image simulation produces virtual images from software representations of imaging devices and virtual object models representing the human body. Object models consist of the geometry of the objects (e.g. organs, tissues, pathological structures, etc.) and of their physical parameters used for the simulation. The diversity of this information makes it difficult to share and reuse across simulation modalities and users. We address this issue by explicitly describing object models using a semantic approach. In particular, we developed an ontology that contains the relevant concepts and relations of the domain of object models for image simulation. This ontology is used to annotate object models and to describe their content and structure. In this paper, we present the construction steps of this ontology, the representation choices and we illustrate how it is used to annotate object models.
\end{abstract}

\section{INTRODUCTION}

Medical image simulation has become an essential tool to improve the understanding of biological processes, pathology diagnosis and treatment. Among others, it is used as ground truth in image processing [1], [2] and to prototype image acquisition methods [3], [4].

Image simulation scenes involve simulators of one or several imaging modalities and an object model representing biological entities or artificial objects such as calibration phantoms. Besides geometry, object models may include dynamic information and must contain modality-specific physical parameters which are usually defined as maps or look-up tables.

In many cases producing image simulations is unwieldy, due to difficulties in installing and mastering simulation software, to the heaviness of computations and to the scarcity of properly parametrized object models. The latter can be addressed by community sharing of object models but this requires a proper data organization. The work presented in this paper is a part of an ongoing project, the Virtual Imaging Platform (VIP), which targets these issues considering simulators of 4 of the main medical imaging modalities, namely Magnetic Resonance Imaging (MRI), Computed Tomography (CT), Positron Emission Tomography (PET) and Ultrasound imaging (US).

Sharing object models requires a suitable representation to store, index and retrieve them. Not only must this representation be able to describe object model characteristics involved in model searching, it also has to provide the information required by the various simulators (e.g. physical characteristics of the tissues).

Our goal is to foster the sharing and reuse of object models by explicitly describing their content and structure. To do that, we develop an ontology used to annotate the models with the relevant domain concepts. As a result, shared object models embed annotations referring to the developed ontology next to the bulk data (set of raw files) representing the object geometry and physical parameters.

In this paper, the semantic object model representation on which these annotations are based is presented. section II details the problem of object model sharing by defining the type of information to be described. section III explains the methodology that was used to build the semantic model and section IV details the ontology content and presents an example of one object model. The paper closes on a discussion about the lessons learned from this semantic modeling.

\section{MODELS FOR MEDICAL IMAGE SIMULATION}

The term model is generic and refers to a wide variety of entities such as physiological models, biological models, geometrical models, etc. Thus, it is difficult to define a common representation adapted to all of them as in FieldML [5]. Other initiatives like the on-going European project Virtual Physiological Human (VPH) [6], also support research in biomedical modeling and simulation of the human body, but do not address the specific problem of medical image simulation. For these reasons, we focus here on data-oriented models dedicated to medical imaging simulation.

These object models convey information related to the object geometry, temporal evolution and physical parameters describing multiple physical qualities of simulated materials. For instance, MR simulation requires proton density and magnetic properties such as $\mathrm{T} 1, \mathrm{~T} 2, \mathrm{~T} 2 *$ and magnetic susceptibility $\chi$. Ultrasound simulation needs echogenicity properties as statistical distributions defining amplitudes and positions of scatterers. For PET simulation, it is necessary to define the radioactivity emitted by tissues and their chemical composition since it determines attenuation properties. In the same way, CT requires chemical composition of tissues. Note that physical parameters sometimes depend on acquisition parameters, e.g. $\mathrm{T} 1$ is related to the main magnetic field strength $\left(B_{0}\right)$ in 
MRI, and potentially on physiology, e.g. the radioactivity in a tumoral zone.

In addition, models contain several kinds of geometrical information related to anatomy, physiology, pathologies, contrast agents and foreign bodies. Various representations of this geometry can be used, the most common being meshes and voxel maps.

Time is another important characteristic since a model can be dynamic and integrate several levels of temporal resolution. For instance, coarse time scales are used in longitudinal follow-up studies while finer time scales describe movement. Multiple time scales can be combined, e.g. in follow-up studies of cardiac pathologies.

Thus, the problem considered here that is to define an object model representation enabling the sharing of object models for multi-modality simulations has multiple facets that must be properly dealt with. Consequently, we decided to use a semantic model that will take into account all the information described above.

\section{BUILDING OF THE SEMANTIC MODEL}

An ontology is a formal specification of entities and their relationships. It provides both a reference vocabulary to refer to entities, as well as the means to formally represents their meanings, using axioms, that can be used to reason and to perform inferences. Among these relationships, is-a and part-of are the most important. Building an ontology is a complex task involving the collaboration of several actors. In this work, we are interested in modeling all the relevant entities which take part in the description of object models for multi-modality image simulation. Emphasis on multi-modality is challenging as it involves modeling common concepts across different user communities. However, this approach is the key to be able to share efficiently models by explicitly describing their content regardless of modality and simulator particularities.

The first step to build the semantic model was to interview and meet with several researchers involved in the use of object models for simulation. We listed model use-cases to highlight the common concepts across modalities and simulation models. We started with a simple representation using list of concepts and a short definition of each concept. Once a consensus was reached amongst the users we started the formal construction of the ontology.

Several methods were proposed as guidelines for the construction of ontologies. A consensual idea among these methods is the concept of modularization, which consists in reusing existing knowledge and in relying on already existing strong foundational references.

At the highest level of our modeling, we decided to rely on the DOLCE [7] (Descriptive Ontology for Linguistic and Cognitive Engineering) foundational ontology, which provides a set of abstract concepts and relationships that are valid across multiple domains. Foundational ontologies provide rigorous frames for ontology development and are helpful to give highlevel categorization of the concepts of a domain. This approach has been successfully used in the past [8] and is recognized as the key to share and map ontologies. Our ontology also relies on a set of core ontologies developed in the frame of NeuroLOG [9], a project aiming at supporting the sharing of data and other resources in neuroimaging. We also used external resources like the Foundational Model of Anatomy (FMA) [10] from which we extracted a set of concepts relevant for our application.

We created a first version of our modeling using the OntoSpec methodology [11] which relies on OntoClean methodology. The representation is semi-formal and describes the concepts and links between them. This representation is independent from the implementation and serves as a reference and documentation of our model. From this OntoSpec specification we created a formal representation in OWL, the web language for ontologies. The OWL representation is less expressive but allows to perform automatic reasoning. We used the Protégé ontology editor ${ }^{1}$ (v4.0) for the development of the OWL version.

\section{RESULTS}

In this section we describe the first version of our semantic modeling for simulation object models. As presented in section II, several kinds of information have to be represented to describe the content and the structure of the object models.

\section{A. Representing object model content}

We started to list the different kinds of object that can be present in an object model. Five different types of object were identified and defined:

- anatomical-object denotes objects relative to anatomical structures (e.g. Brain, Liver) ;

- pathological-object denotes objects relative to pathological structures (e.g. Tumor) ;

- geometrical-object denotes simple geometrical objects (e.g. Sphere), these objects are generally used for test and calibration;

- foreign-body-object denotes foreign body objects that can be present in the model (e.g. Needle) ;

- external-agent-object denotes external agents, such as contrast agents or radiopharmaceuticals used with some modalities (e.g. USPIO).

The entities subsumed by anatomical-object were extracted from the FMA using the vSPARQL [12] query language, which provides useful features to design recursive queries. The pathological-object, geometrical-object, foreign-body-object and external-agent-object were specialized using entities extracted from the NeuroLOG ontologies or from other resources (i.e. the RadLex terminology [13]) as well as entities provided by object model users.

\section{B. Structure of the model in layers}

To represent explicitly the structure of the different objects present in a model, we used the abstract concept of

\footnotetext{
${ }^{1} \mathrm{http}: / /$ protege.stanford.edu
} 


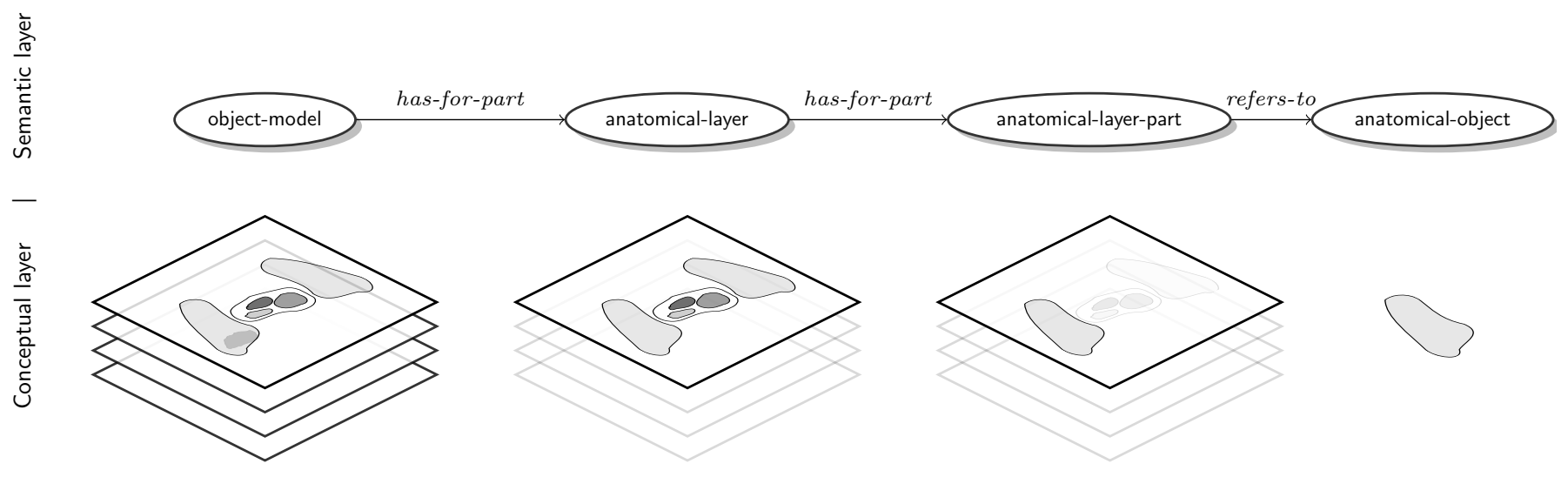

Fig. 1. Model composition in layers. The upper part illustrates the concepts used for the description of the models. The lower part gives a visual representation of the abstract concept of layers. For simplicity the layers are displayed in 2D but they are actually 3D volumes.

object-layer. An object layer gathers all the objects of a given type. Thus, five object layer types exist corresponding to the five types of objects (e.g. anatomical-layer). A layer is itself decomposed into layer-part, each one referring to a unique object (e.g. anatomical-object). Fig. 1 illustrates the layer mechanism and its decomposition into layer-parts. This representation gives a structure to the content of the models and conceptually separates the different types of models according to their content. This representation also eases the conception of models content as the concept of layer is widely used and easy to understand. The layer-part level is useful to store information about the file(s) where the geometry of the object is stored (meshes or voxel masks).

Concerning voxel masks, each layer-part also possesses a label that is an integer value corresponding to the value used to refer to this layer-part in the geometric file. This information is generally extracted from a look-up table (LUT) defining the correspondence between the integer values used in the geometric files, and their meaning in terms of object (e.g., $\{$ Lung $=12$, Heart $=14, \ldots\}$ ).

Concerning the mesh masks, a priority is given to the layerpart through an integer value in order to know how to combine several layer-parts and build a voxel representation before the simulation process.

\section{Static and dynamic models}

The representation in layers allows to model the content and the structure of a model at a given moment in time. However, simulation object models may be static or dynamic. A static-model defines information which does not evolve through time. On the contrary, a dynamic-model reflects the fact that the content of the model can evolve through time.

To describe these evolutions, we used two concepts to represent time: time-point and instant. A timepoint is characterized by a date and is composed of 1 to $n$ instants. Time-points are useful to represent longitudinal-follow-up-model corresponding to models that evolve through long time periods, for example in the case of a longitudinal tumor follow-up study composed

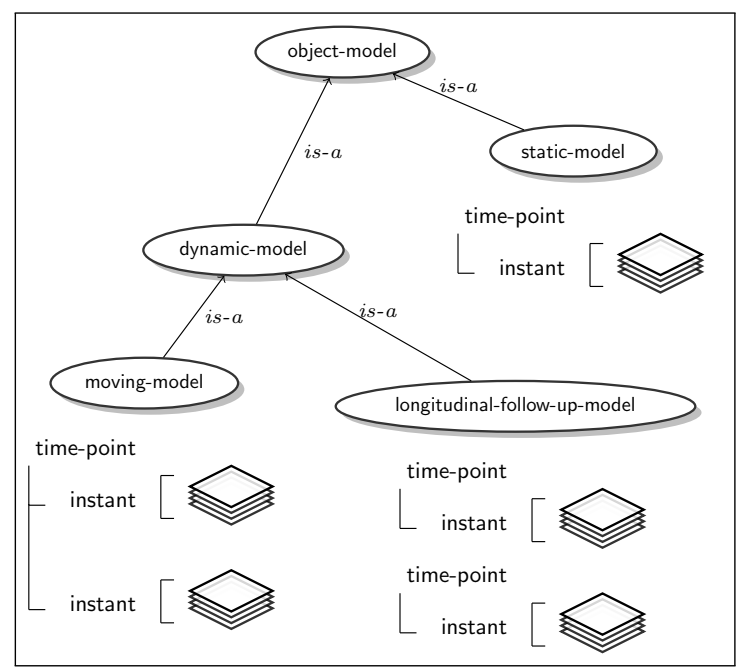

Fig. 2. Use of time-points and instants for static and dynamic models.

of exams of a patient acquired at different days. A time-point is itself composed of instants to represent moving-model, which corresponds to models denoting some physiological process (i.e. respiratory movement, blood flow, etc.). Fig. 2 illustrates the different types of static and dynamic models and their use of instant and time-point.

\section{Representing physical parameters}

Although sharing models containing only the structure and geometry of the objects is already interesting, such models are not sufficient to perform image simulations. Indeed, as mentioned in section II, parameters describing the physical properties of the objects are required by the simulation process. These properties are the chemical composition (for CT and PET), radioactivity (for PET), magnetic properties (for MRI) and echogenicity (for ultrasound). Physical parameters can be described using two representations. The first representation is as a layer (physical-parameters-values-layer) that defines explicitly the values of a given physical parameter at each voxel. The second representation describes the 


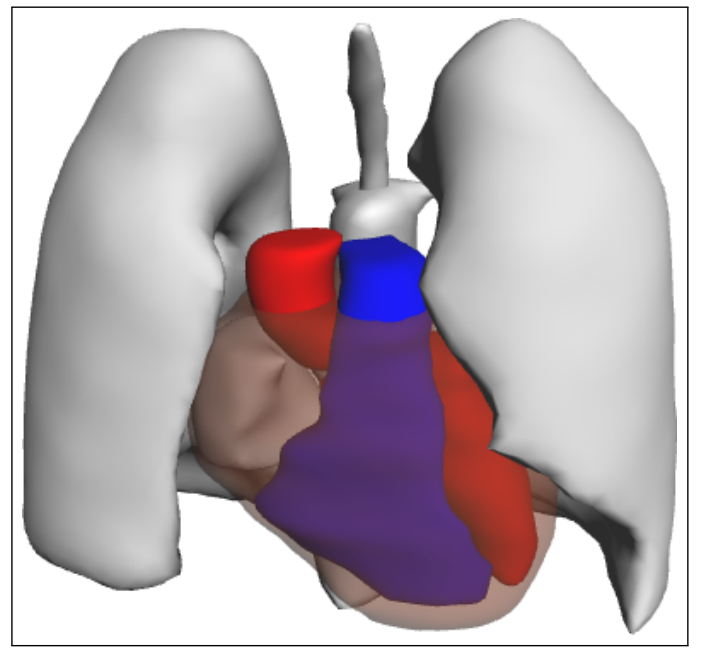

Fig. 3. Mesh representation of one instant of the original ADAM model [14] representing several anatomical objects.

physical parameters of the different objects depicted in the corresponding object layers as probability distributions (e.g. Gaussian) that will be used during the simulation process to compute the actual values at each voxel.

The physical parameter layer can be used in two different cases. First, a physical parameter layer can be linked to an object layer. It means that the physical parameter layer describes the physical properties of the objects defined in the object layer it is linked to. Second, the physical parameters layer can be linked to an instant. In that case, the physical parameter layer describes the information for all the object layers defined at this given instant.

\section{E. Implementation}

In its first OWL version, our ontology is composed of 1500 concepts and 250 relations. We developed a first prototype allowing the creation of instances of models using the developed ontology. Creating an instance of a model consists in creating instances of the different entities defined in our ontology to reflect the content and the structure of a model. We used the Jena ${ }^{2}$ framework to manipulate the ontology and to create instances. We used inference mechanisms to ease the construction and help the user in model annotation (e.g. automatic detection of object type according to their name using the is-a relation). We are at the early stage of the platform development and for the moment no graphic interface is publicly available. However, our prototype already allows us to annotate models. The next section illustrates an example.

\section{F. Illustration}

To validate our semantic approach, we described the ADAM model (illustrated in Fig. 3) using our ontology. We enriched it to have a complete and comprehensive example to illustrate the different concepts. ADAM is a 4D model, i.e. a dynamic 3D model containing 15 instants for one

\footnotetext{
${ }^{2} \mathrm{http}: / /$ jena.sourceforge.net/
}

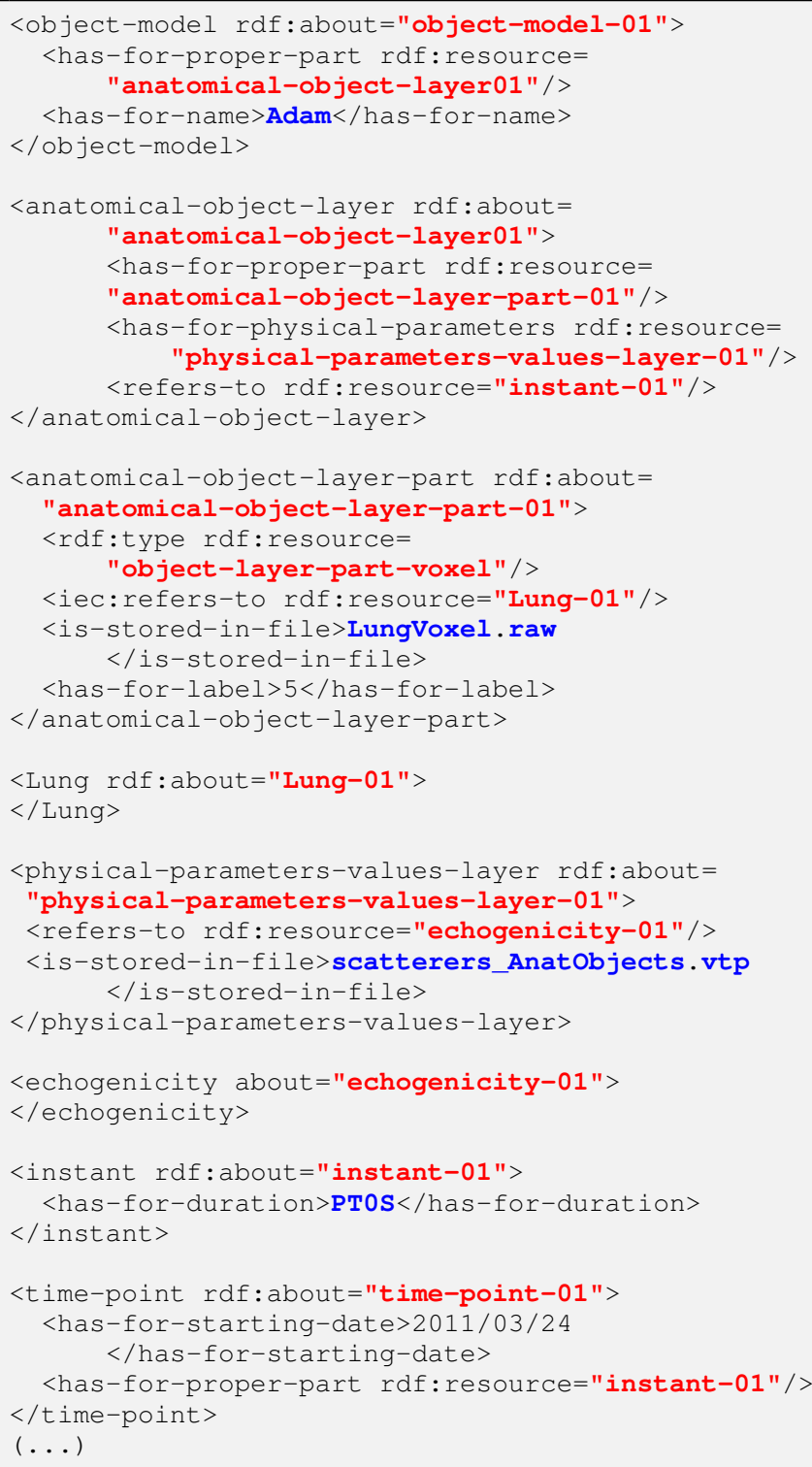

Fig. 4. Example of a subset of annotations of the ADAM model in the RDF format.

cardiac cycle, each defined by mesh and voxelic representation. As explained in section IV-D, a priority level is associated to each mesh and a look-up table associating tissues and matters is associated to each voxel map. The ADAM model is also defined for several time points, corresponding to two different days. Besides, an instant contains geometrical information as an anatomical-object but also a tumor as a pathological-object, a needle as a foreign-body-object and a contrast agent as an external-agent-object. The anatomical-object is composed of several anatomical-layer-part such as Aorta, Ventricles or Lungs. ADAM also contains physical parameters maps and look-up tables linking matters to physical parameters. The data files are bundled in a zip file including an RDF file containing the annotations. A sample of this 
annotation file is shown on Fig. 4.

This annotation file is then manipulated by simulation workflows to adapt the model to the simulator formats. Time points and instants are split to enable data parallelism exploitation on computing platforms. A companion paper about simulation integration in the Virtual Imaging Platform (VIP) describes these workflows in details [15].

\section{Discussion}

In this paper, we presented a semantic approach for representing object models for multi-modality medical image simulation. Object models are complex entities and contain many kinds of information (geometry, physical parameters, types of objects, etc.). Thus, the sharing and the reuse of models is a challenging problem, especially for multi-modality simulation since the models have to carry all the needed information to perform simulation in different modalities. We adopted a semantic approach that consisted in developing an ontology representing the domain of object model for image simulation. This ontology is based on a foundational ontology (DOLCE) and integrates several other resources (NeuroLOG ontologies, FMA, etc.)

The resulting semantic model can describe a broad range of models and can explicitly annotate their content. It can also be used to adapt object models to simulators as described in [15]. By using common concepts defined and described in our ontology, we ensure the interoperability and the efficient sharing of the models. Semantic querying will enable to extend the query capabilities thanks to the use of explicit knowledge embedded in the ontology. This semantic approach will also be used to automate the creation of the annotations of the images produced using the object models in simulation processes. Indeed, medical image annotation using ontologies has received a strong interest to improve the semantic description of images content. However, manual annotation is a tedious task, and the use of annotated models will allow us to perform this task automatically.

Our work has to be put in perspective with other works aiming at sharing biosimulation models, such as cardiovascular anatomical models [16] or other kinds of models e.g. developed in the context of the VPH project - modeling physiology or pathology and their relation to anatomy [17]. All of these initiatives share the same concern of disambiguating the complex features of those models, as well as the concern of enabling model composition and interoperability, and therefore they rely on ontologies to provide the sound and formal definitions that are needed. Of course, medical image simulation object models address a specific family of these models, characterized by a prominent relation to biological objects' geometry and to their physical characteristics, but it is of key importance that such characteristics may be derived in the future from other general-purpose biosimulation models. So, special efforts will be devoted toward achieving such interoperability.

The next step of our work is to develop the platform which will be used for the interactive annotation of object models.
We are also working on improving the ontology by studying other use-cases involving object models in order to broaden the scope of our work and assess the relevance of our semantic model.

\section{ACKNOWLEDGMENT}

We gratefully thank all the participants of the "GDR STIC Santé" January 2011 workshop for their useful input and feedback on this topic. This work is funded by the French National Agency for Research under grant ANR-09-COSI-03 "VIP".

\section{REFERENCES}

[1] C. Lartizien, S. Marache, A. Le Pogam, S. Tomei, and D. Visvikis, "Impact of partial volume correction in whole-body PET imaging : a computer-aided detection study," in IEEE NSS and MIC Conference, Orlando, USA, October 2009, pp. in-press.

[2] P. Bromiley and N. Thacker, "Multi-dimensional medical image segmentation with partial volume and gradient modelling," Annals of the British Machine Vision Association and Society for Pattern Recognition, Tech. Rep. 2, 2008.

[3] H. Liebgott, A. Basarab, P. Gueth, D. Friboulet, and P. Delachartre, "Transverse oscillations for tissue motion estimation," Ultrasonics, vol. 50, no. 6, pp. 548-555, 2010.

[4] O. Addy, D. Charpigny, M. Sigovan, E. Canet-Soulas, H. Benoit-Cattin, and D. Nishimura, "MRI simulation framework for atherosclerosis inflammation with USPIOs," in European Society for Molecular Imaging, 2009.

[5] G. R. Christie, P. M. Nielsen, S. A. Blacket, C. P. Bradley, and P. J. Hunter, "Fieldml: concepts and implementation," Phil. Trans. R. Soc. A, vol. 367, no. 1895, pp. 1869-1884, 2009.

[6] M. Viceconti, G. Clapworthy, and J. Van Sint, "The Virtual Physiological Human-a European initiative for in silico human modelling." The journal of physiological sciences: JPS, vol. 58, no. 7, p. 441, 2008.

[7] C. Masolo, S. Borgo, A. Gangemi, N. Guarino, A. Oltramari, R. Oltramari, L. Schneider, L. P. Istc cnr, and I. Horrocks, "The wonderweb library of foundational ontologies and the DOLCE ontology. Wonderweb deliverable D18, final report (v. 1.0, 31-12-2003)," 2003.

[8] L. Temal, M. Dojat, G. Kassel, and B. Gibaud, "Towards an ontology for sharing medical images and regions of interest in neuroimaging," Journal of Biomedical Informatics, vol. 41, pp. 766-778, October 2008.

[9] M. Dojat, M. Pélégrini-Issac, F. Ahmad, C. Barillot, B. Batrancourt, A. Gaignard, B. Gibaud, P. Girard, D. Godard, G. Kassel, D. Lingrand, G. Malandain, F. Michel, J. Montagnat, X. Pennec, J. Rojas Balderrama, and B. Wali, "NeuroLOG: A framework for the sharing and reuse of distributed tools and data in neuroimaging," in Organization for Human Brain (OHB'11), Québec city, Canada, 2011.

[10] C. Rosse and J. L. V. Mejino, "The foundational model of anatomy ontology," in Anatomy Ontologies for Bioinformatics, ser. Computational Biology, A. Burger, D. Davidson, and R. Baldock, Eds., 2008, vol. 6, pp. 59-117.

[11] K. G., "Integration of the DOLCE top-level ontology into the OntoSpec methodology, LaRIA research report 2005-2008," 2003.

[12] M. Shaw, L. T. Detwiler, N. Noy, J. Brinkley, and D. Suciu, "vSPARQL: A view definition language for the semantic web," Journal of Biomedical Informatics, vol. In Press, 2011.

[13] S. Kundu, M. Itkin, D. A. Gervais, V. N. Krishnamurthy, M. J. Wallace, J. F. Cardella, D. L. Rubin, and C. P. Langlotz, "The IR Radlex project: An Interventional Radiology Lexicon-a collaborative project of the Radiological Society of North America and the Society of Interventional Radiology," Journal of Vascular and Interventional Radiology, vol. 20, no. 4, pp. $433-435,2009$.

[14] R. Haddad, P. Clarysse, M. Orkisz, D. Revel, and I. Magnin, "A realistic anthropomorphic numerical model of the beating heart," Functional Imaging and Modeling of the Heart, vol. 26, no. 4, pp. 270-272, 2005.

[15] A. Marion, G. Forestier, H. Benoit-Cattin, S. Camarasu-Pop, P. Clarysse, R. F. da Silva, B. Gibaud, T. Glatard, P. Hugonnard, C. Lartizien, H. Liebgott, J. Tabary, S. Valette, and D. Friboulet, "Multi-modality medical image simulation of biological models with the Virtual Imaging Platform (VIP)," in IEEE CBMS 2011, Bristol, UK, 2011. 
[16] D. Gianni, S. McKeever, T. Yu, R. Britten, H. Delingette, A. Frangi, P. Hunter, and N. Smith, "Sharing and reusing cardiovascular anatomical models over the Web: a step towards the implementation of the virtual physiological human project." Philosophical transactions. Series A, Mathematical, physical, and engineering sciences, vol. 368, no. 1921, pp. 3039-3056, 2010.
[17] J. H. Gennari, M. L. Neal, M. Galdzicki, and D. L. Cook, "Multiple ontologies in action: Composite annotations for biosimulation models," Journal of Biomedical Informatics, vol. 44, no. 1, pp. 146 - 154, 2011, ontologies for Clinical and Translational Research. 